\section{Group N Streptococci}

THe question of the identity of Streptococcus lactis, important in milk and dairy products, has for many years interested bacteriologists. Many are convinced that it is identical with, or a mere variety of, Str. faccalis. With the advent of the Lancefield technique, it has been possible to differentiate into well-defined serological groups certain types of streptococci, and Groups $A$ to $M$ have been thus defined. Str. foecalis falls into Group $D$ of Lancefield.

In $1936^{1}$ we prepared group specific sera for Str. lactis and found that serologically it was quite distinct from Str. foecalis. We have now been able to complete this work and to show that Str. lactis is a member of a new group, to which Str. cremoris also belongs, which we propose to call Group $N$.

Recently Sherman, Smiley and Niven ${ }^{2}$ and Seeleman and Nottbohm ${ }^{3}$ have also established the serological identity of Str. lactis, and the latter authors attached to it the group letter $L$. But letters $L$ and $M$ have already been assigned by $\mathrm{Fry}^{4}$ to streptococci isolated from dogs. Since we appear to have been the first to prepare specific group sera for Str. lactis we may perhaps claim the right to propose the group letter $N$. It would be unfortunate if confusion in nomenclature should invade the serological groups of the streptococei. We have therefore directed attention to the present position, which no doubt may be attributed to the lack of contact between workers engendered by the War.

The results of our work, which deals more fully with the organisms falling into Group $D$ in their relationship to Group $N$, will be published shortly.

A. T. R. MatTick.

National Institute for Research

in Dairying,

University, Reading.

Feb. 11.

${ }^{1}$ Rep. Nat. Inst. Res. Dairy., Reading, 37 (1937).

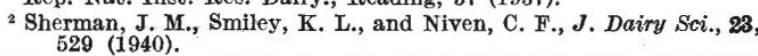

${ }^{3}$ Seeleman, M., and Nottbohm, H., Zbl. Bakt. I. Orig., 146, 142 (1940). ${ }^{4}$ Fry, R. M. Paper read at meeting of Pathological Section of Royal

\section{Auxin Production during Development of the Grain in Cereals}

IT has been shown by Hatcher and Gregory ${ }^{1}$ that auxin accumulates in the developing rye grain from the third week after anthesis, but almost entirely disappears ás the grain ripens. These experiments were continued in 1942 to extend our knowledge of the auxin relations of the grain. The results here briefly discussed will be presented in detail elsewhere.

Avery, Berger and Shalucha ${ }^{2}$ found that the yield of auxin from dry maize grains is increased tenfold by extracting in an alkaline medium of $p \mathrm{H} 10$. Using the same technique with rye, twenty times as much auxin was obtained as with water. It was evident that the dry grain contains only a small amount of auxin but a large amount of an inactive substance from which auxin is formed by heating in alkaline solution. In 1942 both methods of extraction, with and without alkaline hydrolysis, were systematically compared, and no differences in extracted auxin were obtained until after the attainment by the grain of maximum auxin content. The appearance of the inactive substance is thus concurrent with the disappearance of the auxin, and there is little doubt that the one is formed from the other. The inactive substance in rye can scarcely be a precursor of auxin, but is rather an auxin derivative. Isolating the grain was found not to prevent auxin disappearance, as would be the case were auxin normally translocated away from the grain, and this is additional evidence of its inactivation in situ.

In maize, however, the situation seems to be quite different. Avery, Berger and Shalucha ${ }^{3}$ determined the auxin content of maize grains at certain stages in their development and found that there is always an overwhelming predominance of inactive substance over free auxin, indeed as much as 97 per cent of their total at the stage of maximum auxin content. At the same stage in rye there is no inactive substance, and this difference between the two cereals is noteworthy.

In an attempt to locate the site of auxin formation in rye the whole ear was analysed. The results show that auxin first appears in the ear as it emerges, but is confined to the green anthers. This auxin disappears as the anthers ripen, but unlike that of the grain cannot be recovered by alkaline hydrolysis. Subsequent to anthesis no auxin was found except in the carpel, suggesting that it is formed in situ. More precise location of the auxin was made by extracting separately the excised embryos, the aleurone and the endosperm. This revealed that the auxin is absent from the embryo, and is confined mainly to the aleurone layer. Further it was found, by extracting transverse sections of the whole grain, that the auxin is much more concentrated at the embryo end. It may thus be concluded that the auxin is formed inside the grain, in the aleurone region near the embryo.

There is evidence to show that the auxin in the rye grain, which accumulates relatively late in development, is not derived by translocation to the ear since premature harvesting in no way interferes with auxin production. That auxin is not produced directly from the products of assimilation is shown by the fact that accumulation proceeds in ears kept in total darkness from a stage prior to its normal first appearance. Accumulation begins at a definite stage in ontogeny, and it is interesting to note that Nutman $^{4}$ postulates the formation of hormone in certain tissues of the rye ovary which suffer degeneration during normal development. These include the endosperm adjacent to the embryo, which is the region of auxin accumulation. Premature harvesting does not curtail the developmental changes, as Nut$\operatorname{man}^{5}$ has shown, but though in the dwarf grains so produced starch accumulation in the endosperm is practically absent, the aleurone layer is well developed. In these dwarf grains auxin has been shown to occur in high concentration. As to the immediate source of the auxin, I am inclined to the view that it is derived from the cytoplasm of the disintegrating cells, and though the auxin of the rye grain has not yet been identified, it may prove to be indole-3acetic acid, as in maize ${ }^{6}$.

The ear of rye is thus characterized by two separate systems of auxin production, one in the developing anther, the other in the developing carpel. In both systems auxin first accumulates and then disappears, but whereas in the carpel an inactive derivative is formed from which auxin can be recovered by alkaline hydrolysis, in the case of the anther there is no evidence of the same reversibility. 